\title{
In-flight validation of SPEX aifborne spectro-polarimeter onboard NASA's research aircraft ER-2
}

J. M. Smita, Jeroen H. H. Rjetjensa, A, dj Nojaj,

O.P. Hasekampa, W. Laauwen ${ }^{b}$, B. Cajirnsb,

B. van Diedenhoven ${ }^{b}, A$, Wasilewskjb

(a)SRON Netherlands Institute for Space Research,

(b)NASA Goddard Institute for Space Studies 


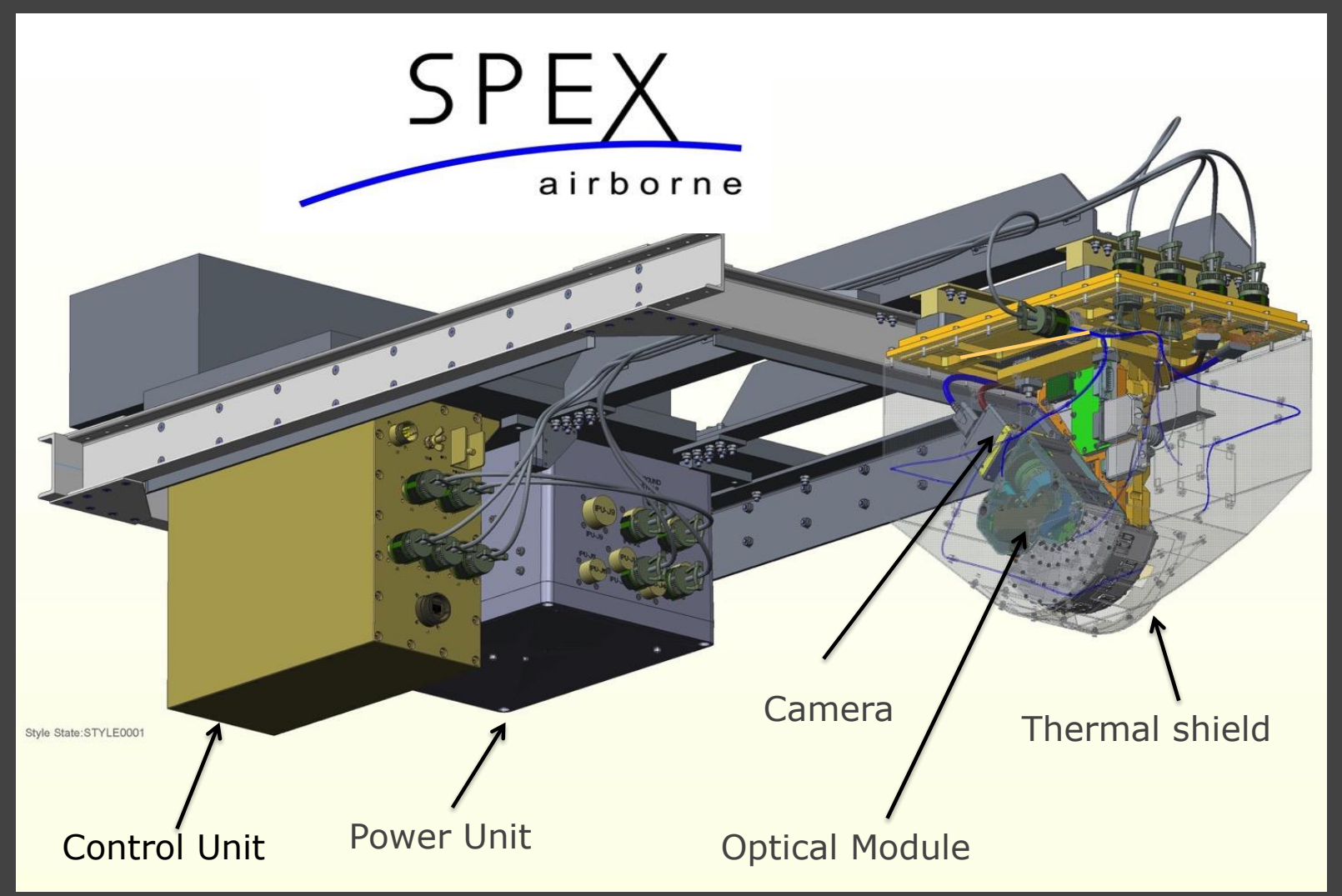

\section{SRON}

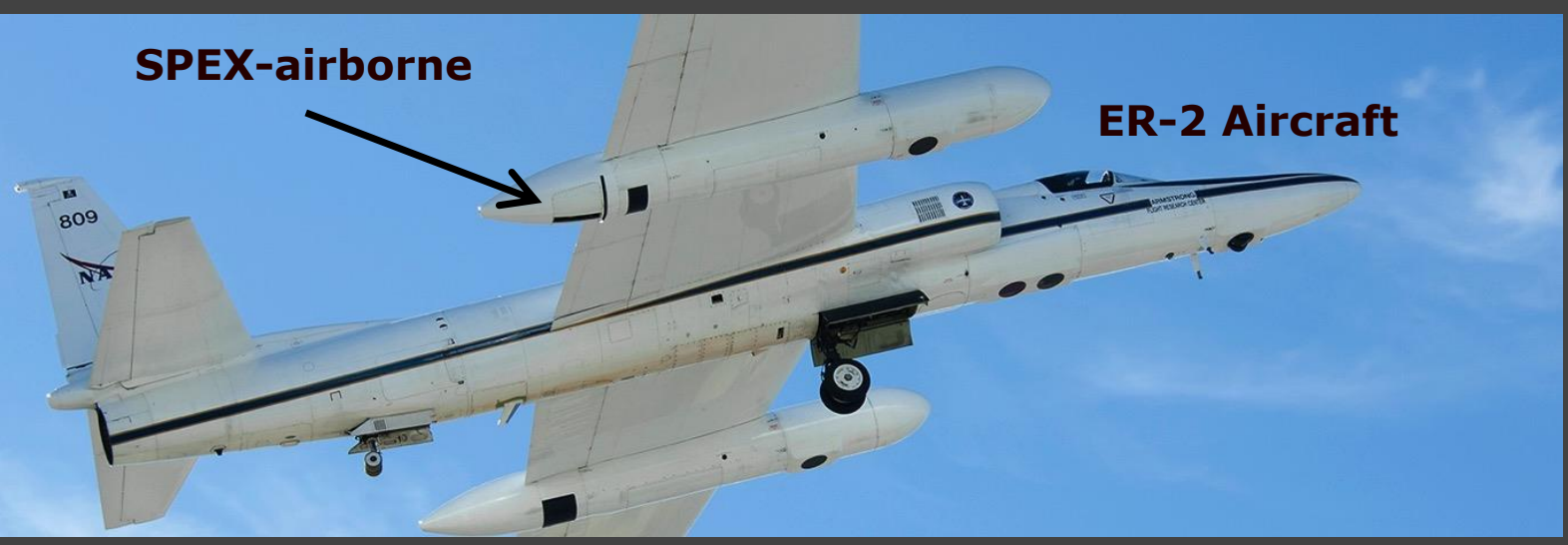




\section{SPEX airborne specifications}

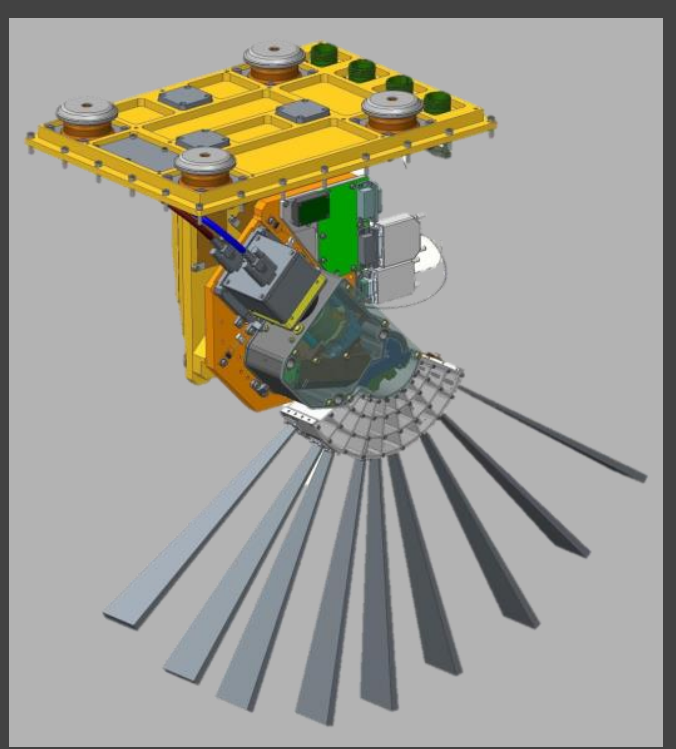

SRON
- Range: 400-800nm

- Spectral resolution radiance: $3 \mathrm{~nm}$

- Spectral resolution DoLP: 7-30 nm

- 9 fixed viewing angles at $00^{\circ}, \pm 14^{\circ}$, $\pm 28^{\circ}, \pm 56^{\circ}$,

- Swath: 70

- Resolution: $1^{0} \times 10\left(350 \times 350 \mathrm{~m}^{2}\right)$

- Sample res: $0.2 \times 10\left(70 \times 350 \mathrm{~m}^{2}\right)$

- Frame rate: $25 \mathrm{~Hz}$ (44 co-added)

- Image: $1 / 1.75 \mathrm{~Hz}$

- Accuracy:

- Accuracy DoLP: 0.002

- Accuracy Radiance: $4 \%$

- Optical module by TNO, ASTRON-NOVA, AIRBUS-NL, SRON \& NL space companies 


\section{Spectral polarization modulation}

- Highly accurate polarimetry

- Snapshot Radiance + DoLP

- Dual beam implementation

- Robust

- Passive

Achromatic quarter A-thermal multiplewave retarder order retarder
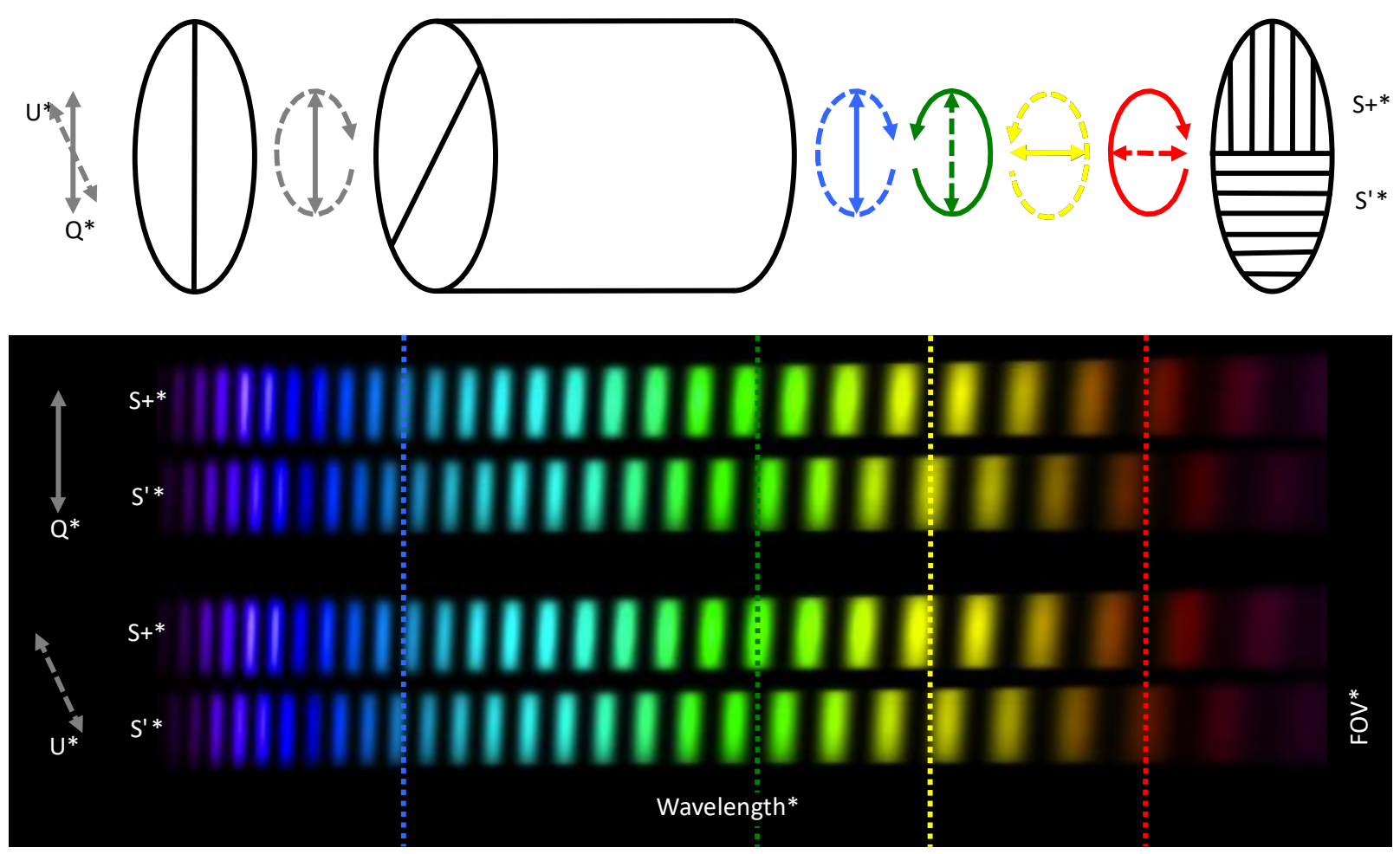

Snik et al., Applied Optics, 2009 


\section{Instrumental polarization calibration}

Polarization State Generator-2

For AirMSPI polarimeter JPL

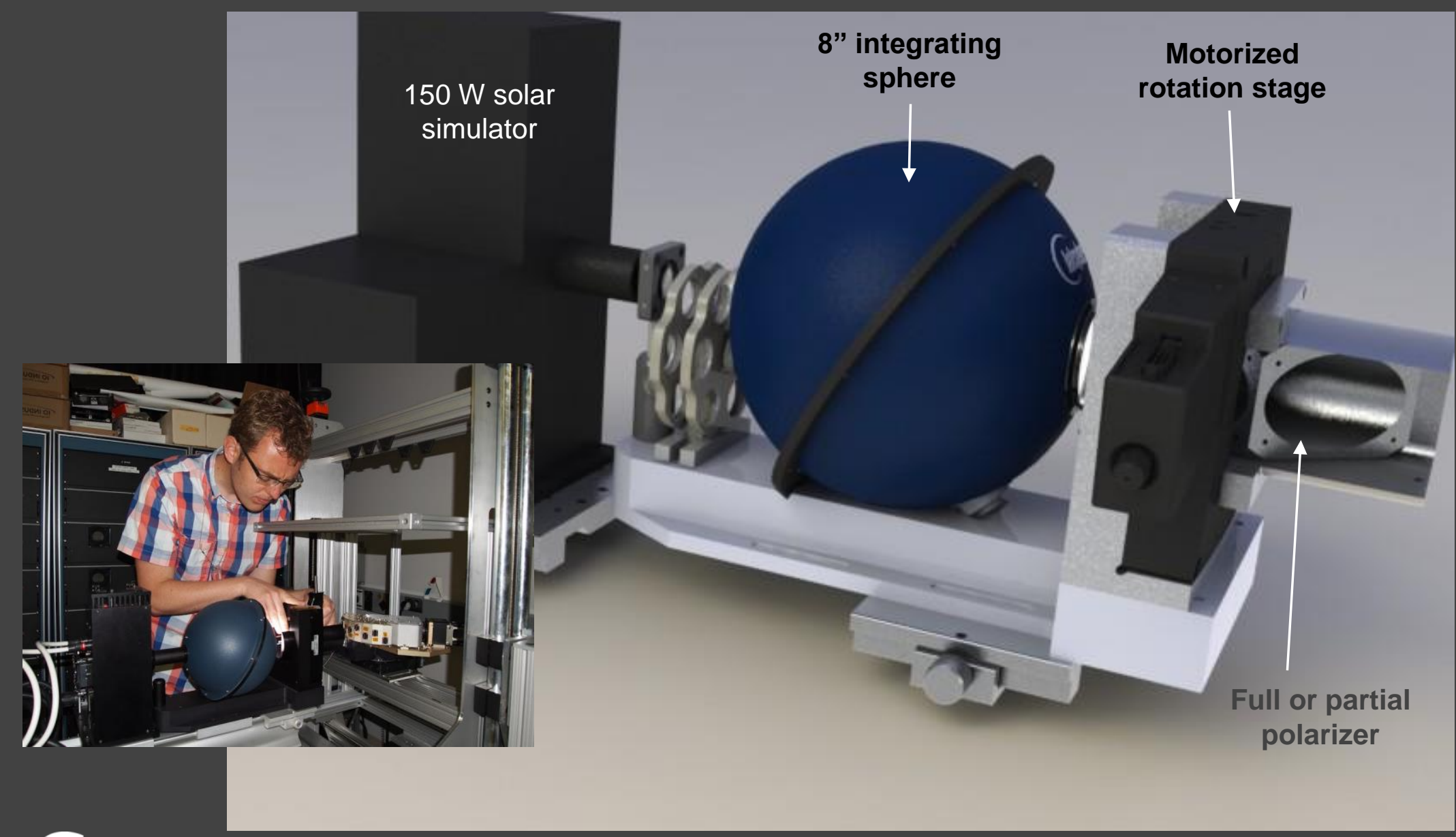




\section{Calibration using $100 \%$ polarized light}

Integrating

sphere

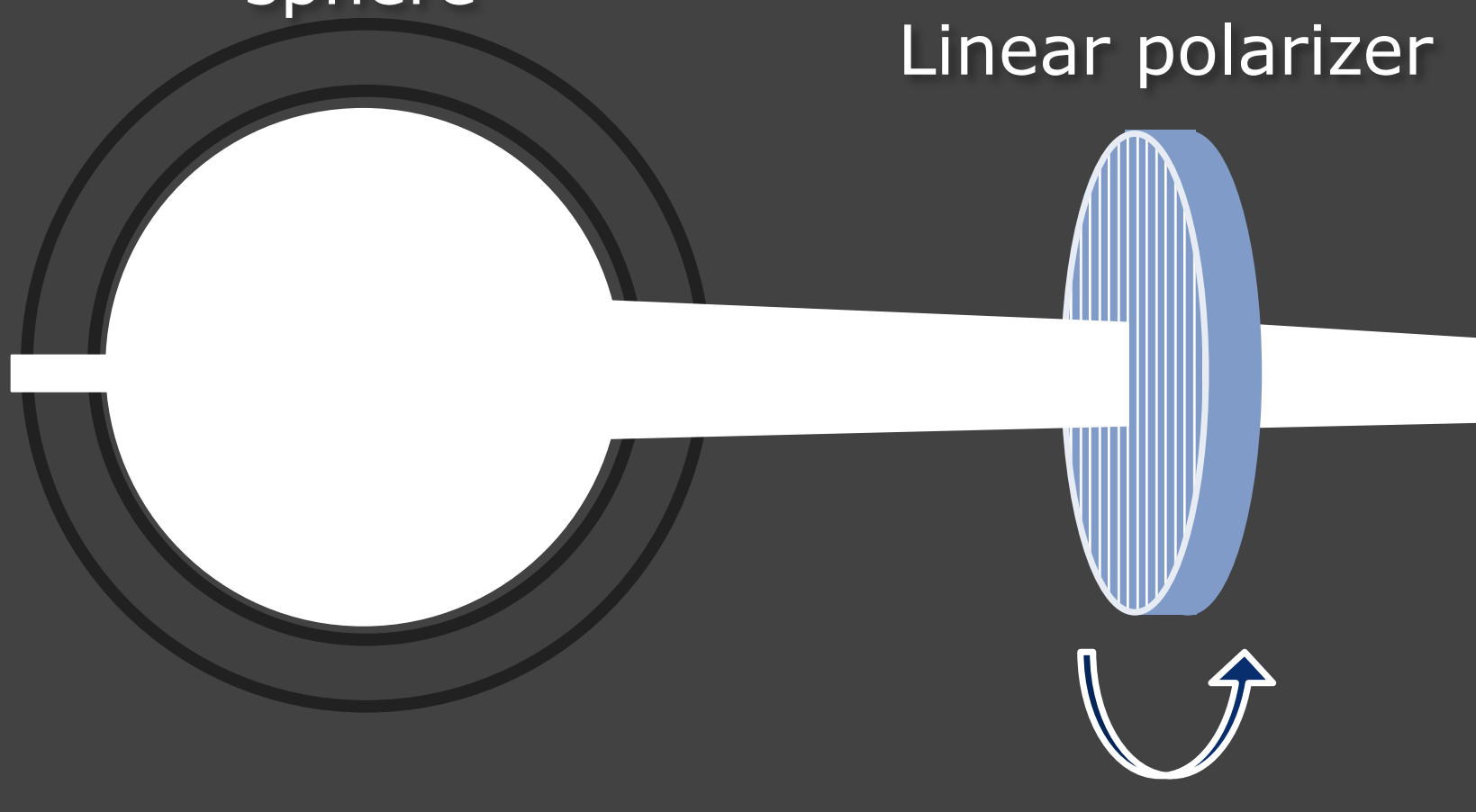




\section{SPEX airborne lab-performance}

\section{Calibration at three "airMSPI" wavelengths: 470, 555, $660 \mathrm{~nm}$}
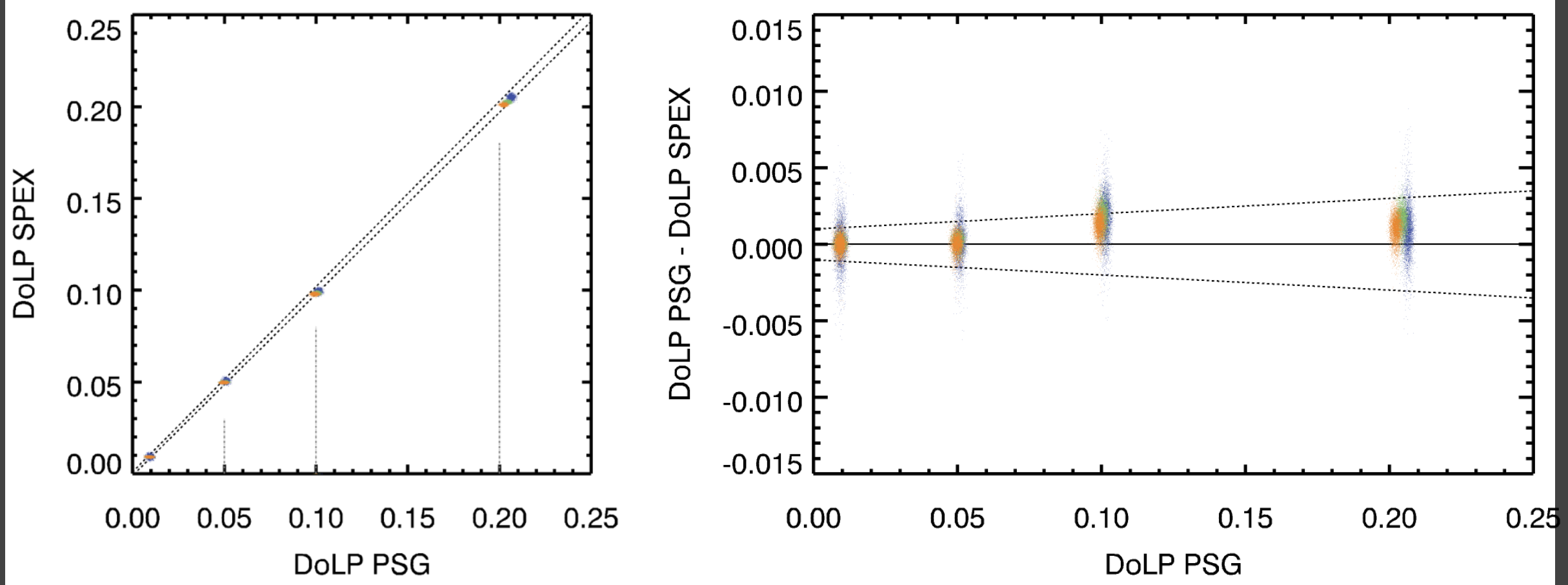

Polarimetric Performance : $\triangle \mathrm{DoLP}<0.002$ 


\section{ACEPOL: Aerosol Characterization from Polarimeters and Lidars}

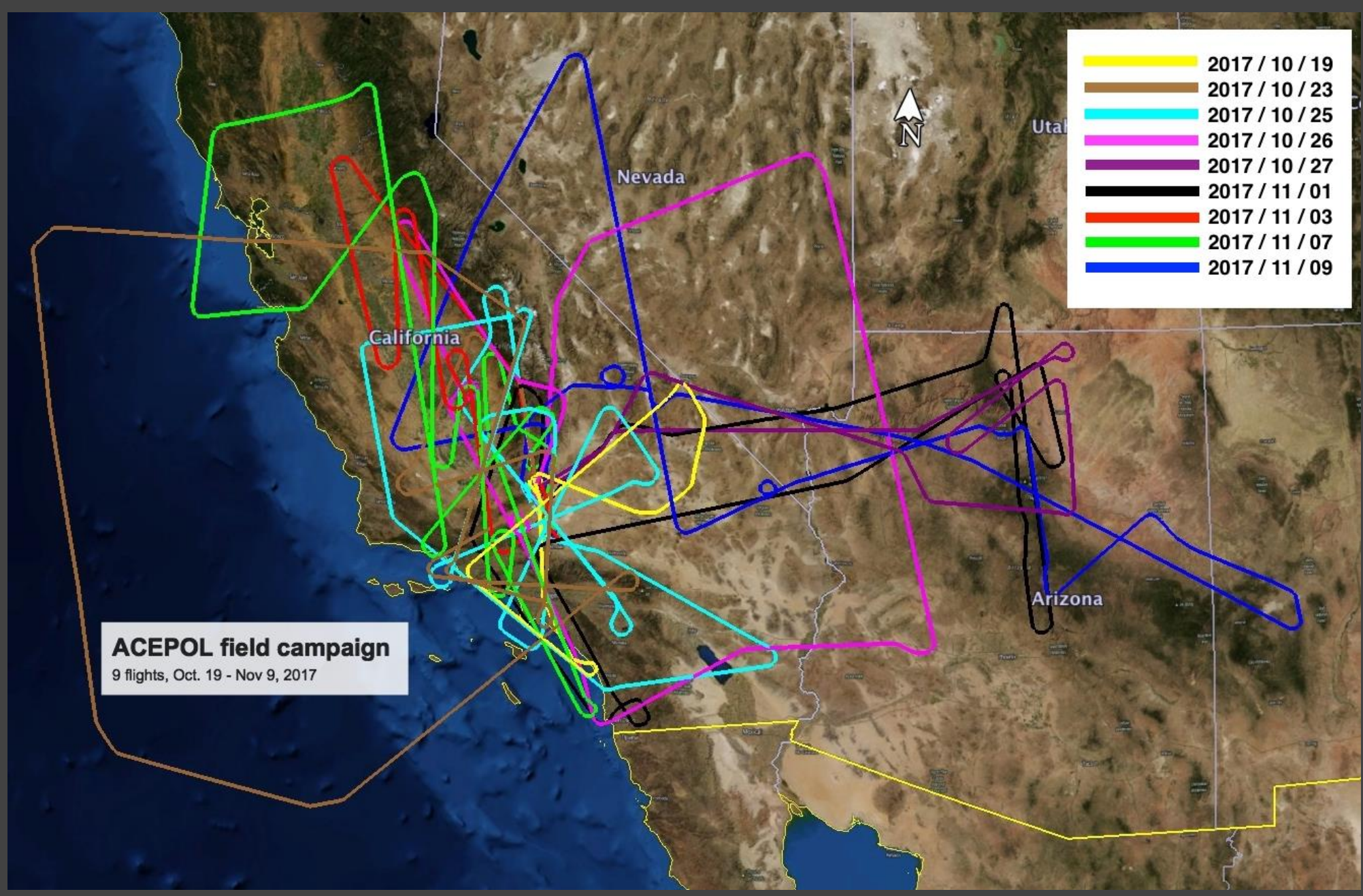

9 flights, 41 hours 


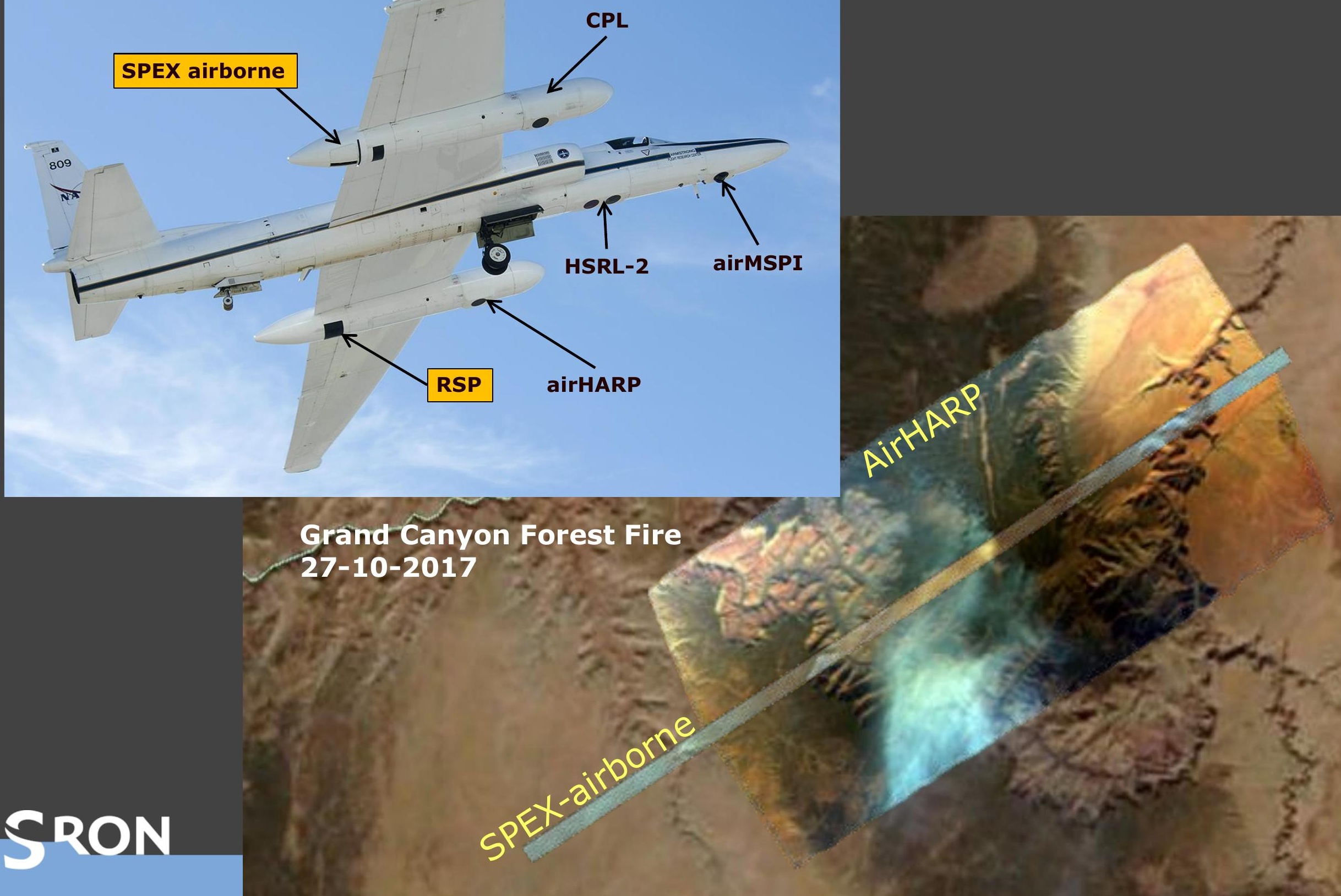




\section{SPEX airborne observations sample}

Modesto California

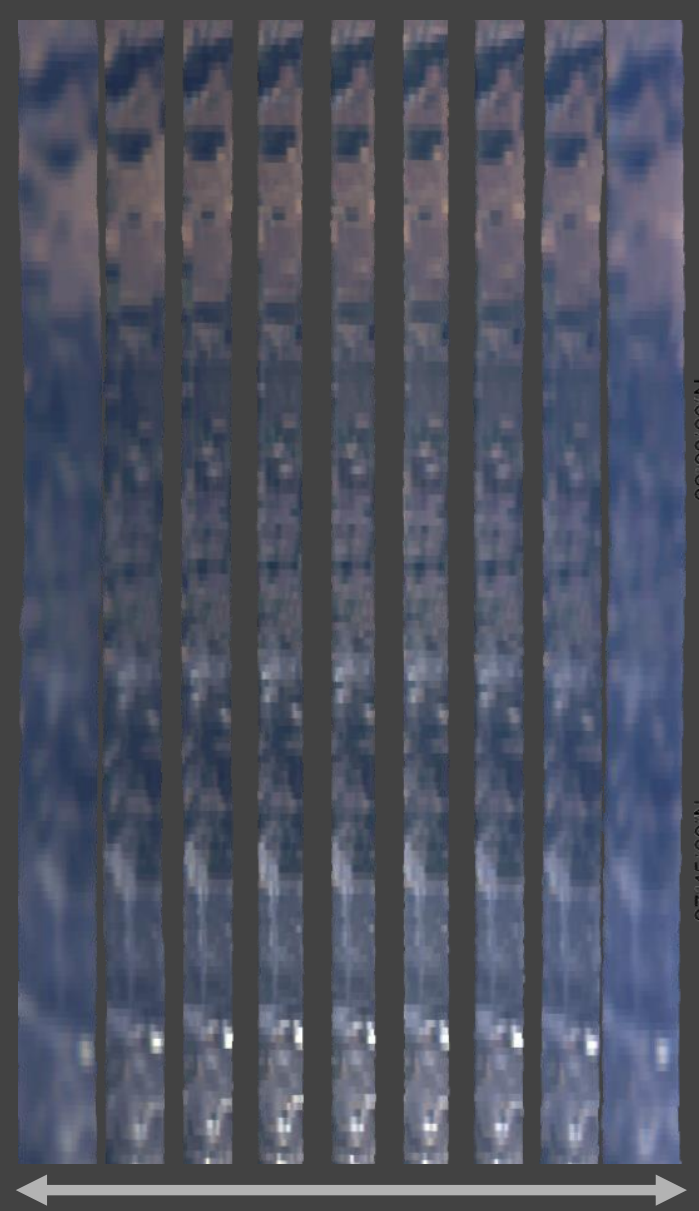

Viewing angle

Nine pushbroom images
Fresno area California

Radiance Polarization

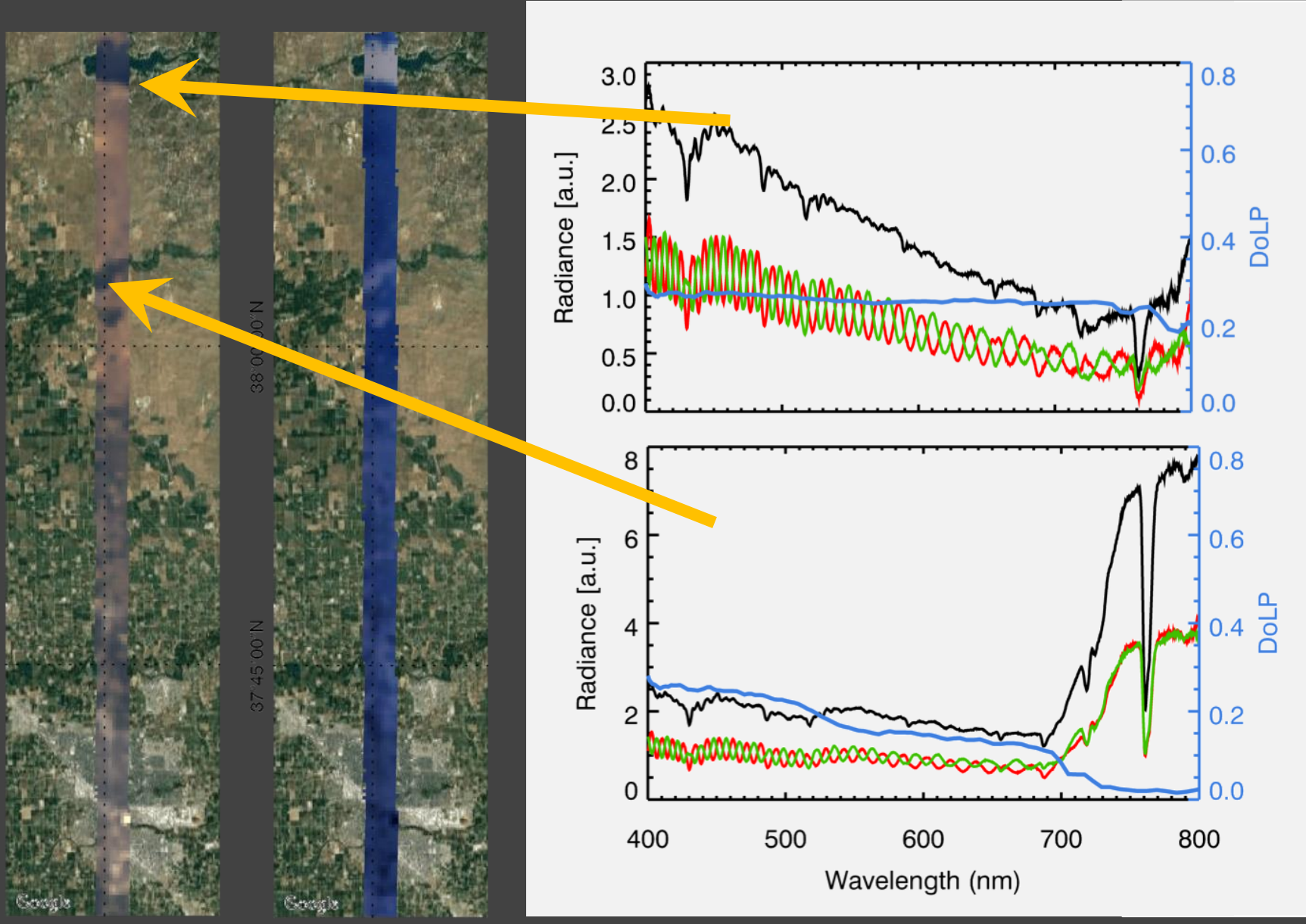




\section{Research Scanning Polarimeter}

- Prototype for Aerosol Polarimetry Sensor on Glory mission (launch failed 2011)

- Simultaneous measurements of Stokes parameters $I, Q$ and $U$

- Polarimetry using polarizing beam splitters

- Scan-mirror system provides continuous sampling of viewing angle

- Single field of view $14 \mathrm{mrad}\left(0.8^{\circ}\right)$

- 152 along-track angles over $120^{\circ}$

- 9 bands in visible and shortwave infrared:

- 410, 470, 555, 670, 864, 960, $1593,1880,2263 \mathrm{~nm}$

- Accuracy:

Polarimetric $<0.005$, radiometric $<5 \%$

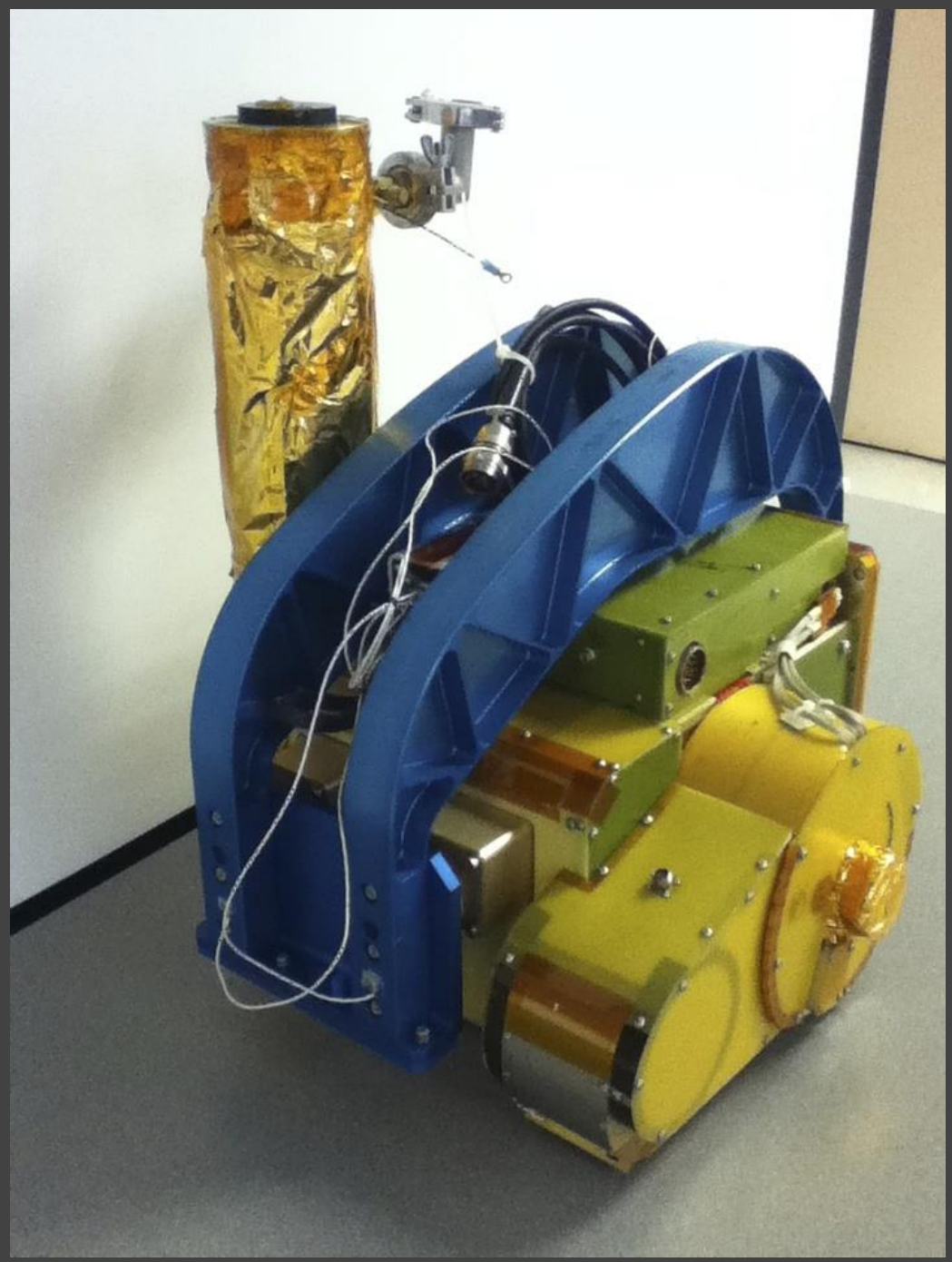




\section{Data comparison strategy}

- Collect data pairs SPEX airborne \& RSP

- Coincident in

- Wavelength

- Time

- Viewing direction $\}$ Geolocation
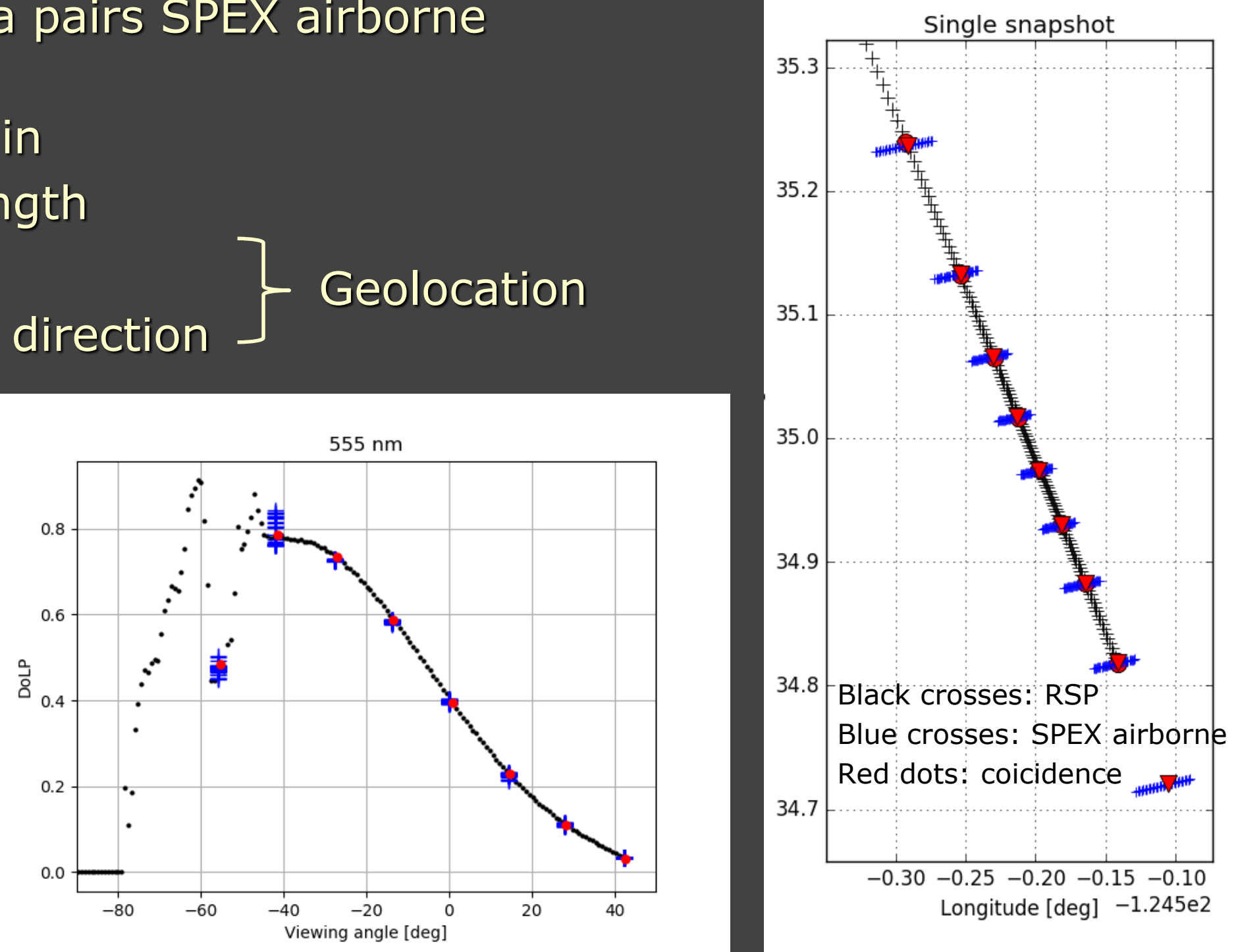


\section{San Francisco Bay area}

Radiance $670.0 \mathrm{~nm}$. Track 20171023-191750. Viewport 4, angle $42.4 \mathrm{deg}$, RSP angle 42.7

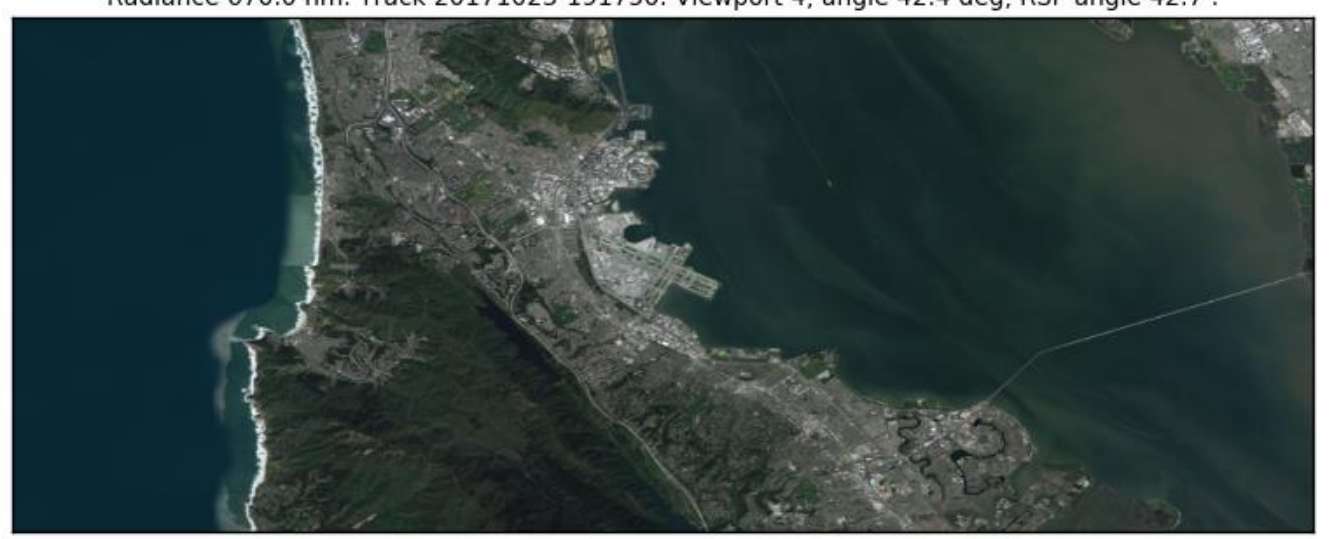

SRON 


\section{San Francisco Bay area. SPEX VP $+42^{\circ}, 670 \mathrm{~nm}$}

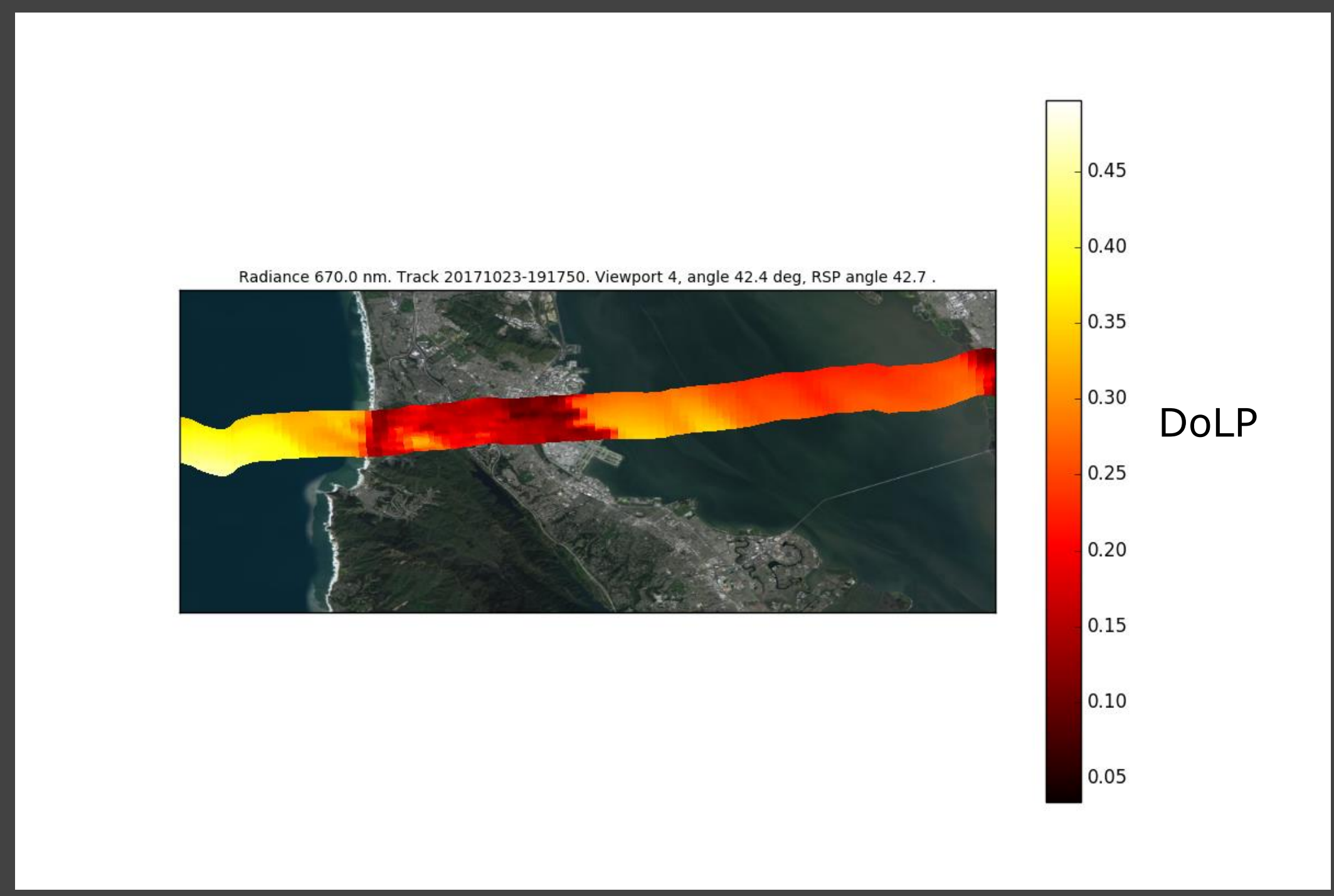




\section{San Francisco Bay area. RSP VP $+42^{\circ}, 670 \mathrm{~nm}$}

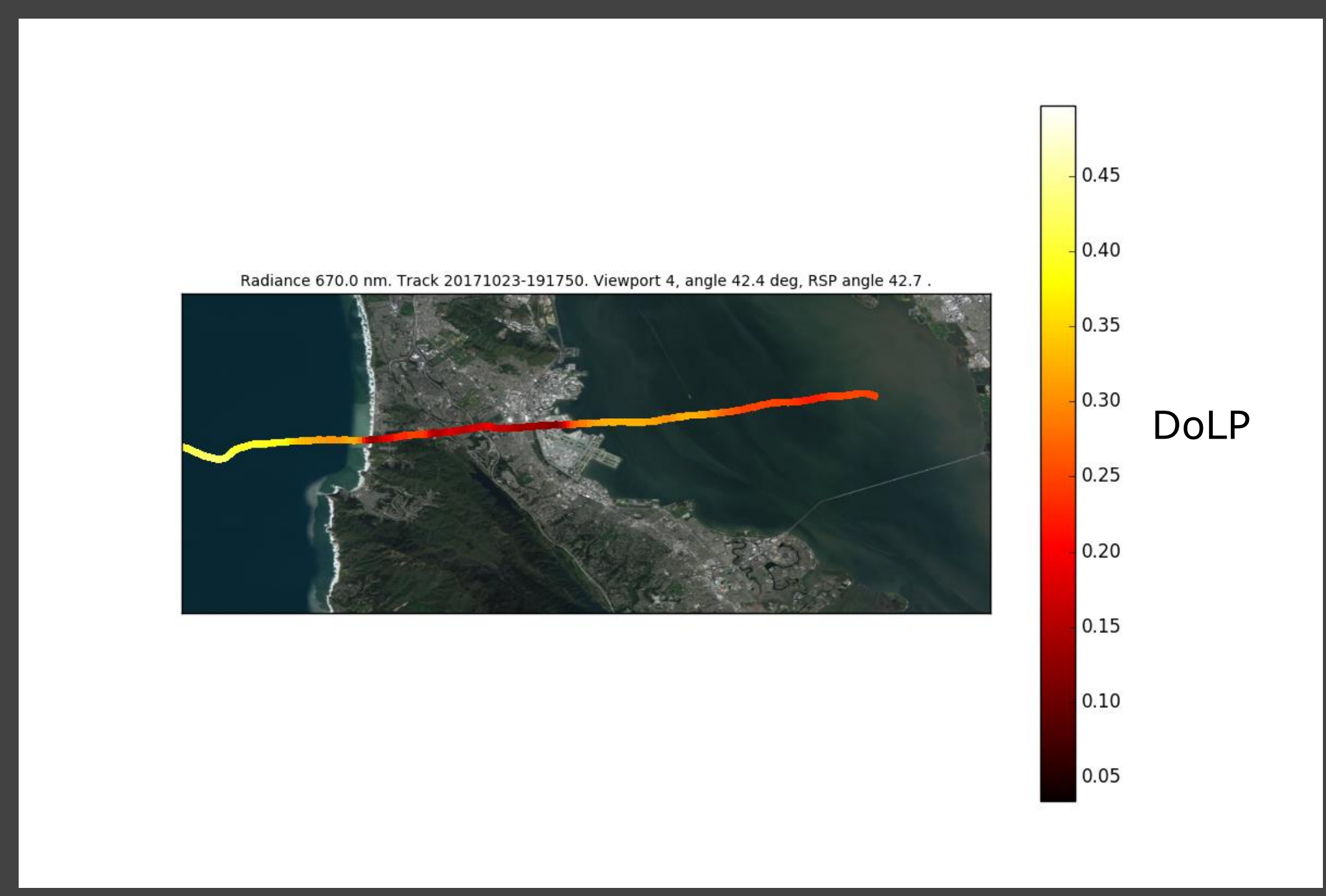

\section{SRON}




\section{San Francisco Bay area. RSP VP $+42^{\circ}, 670 \mathrm{~nm}$}

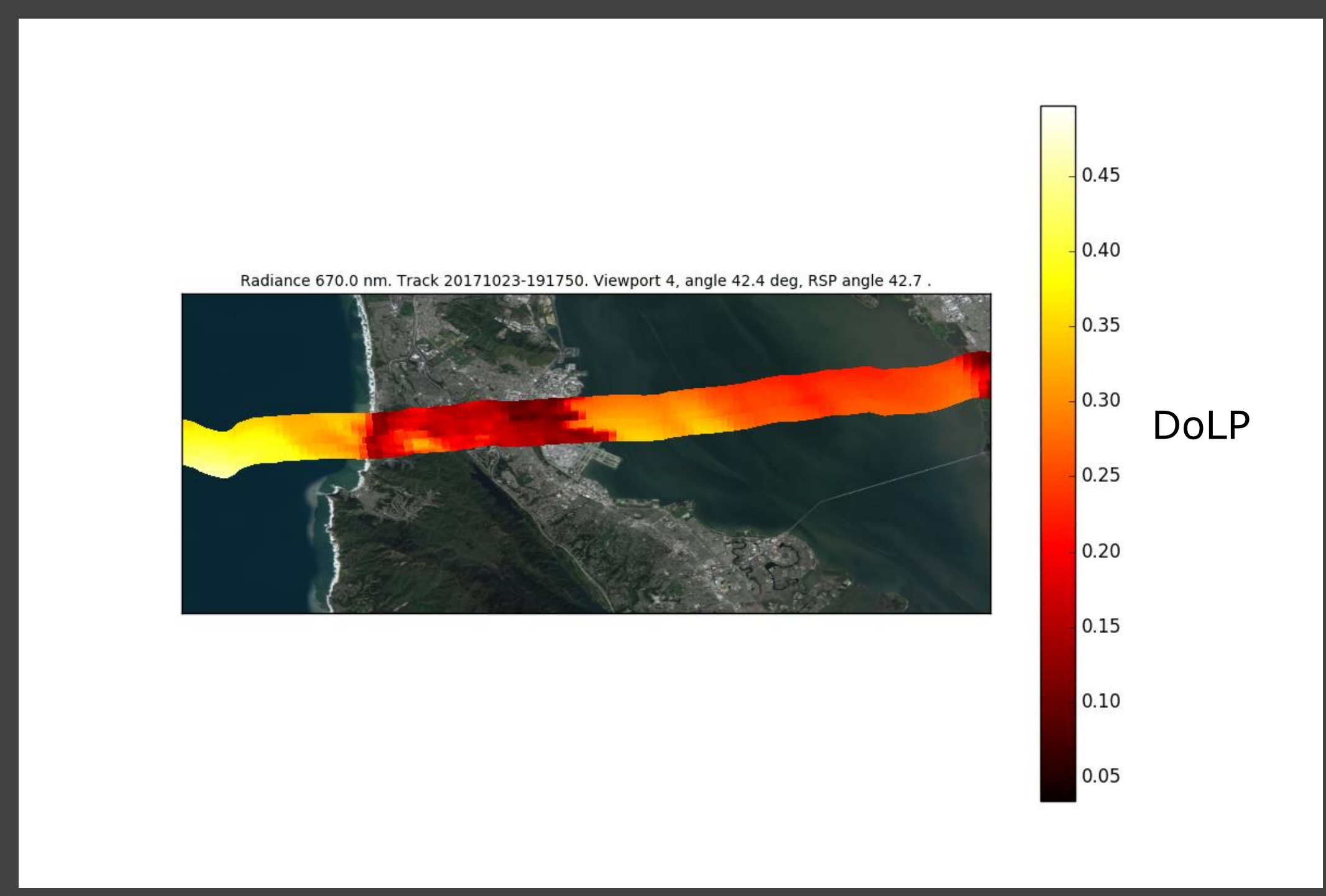

\section{SRON}




\section{SPEX airborne \& RSP over Ocean}
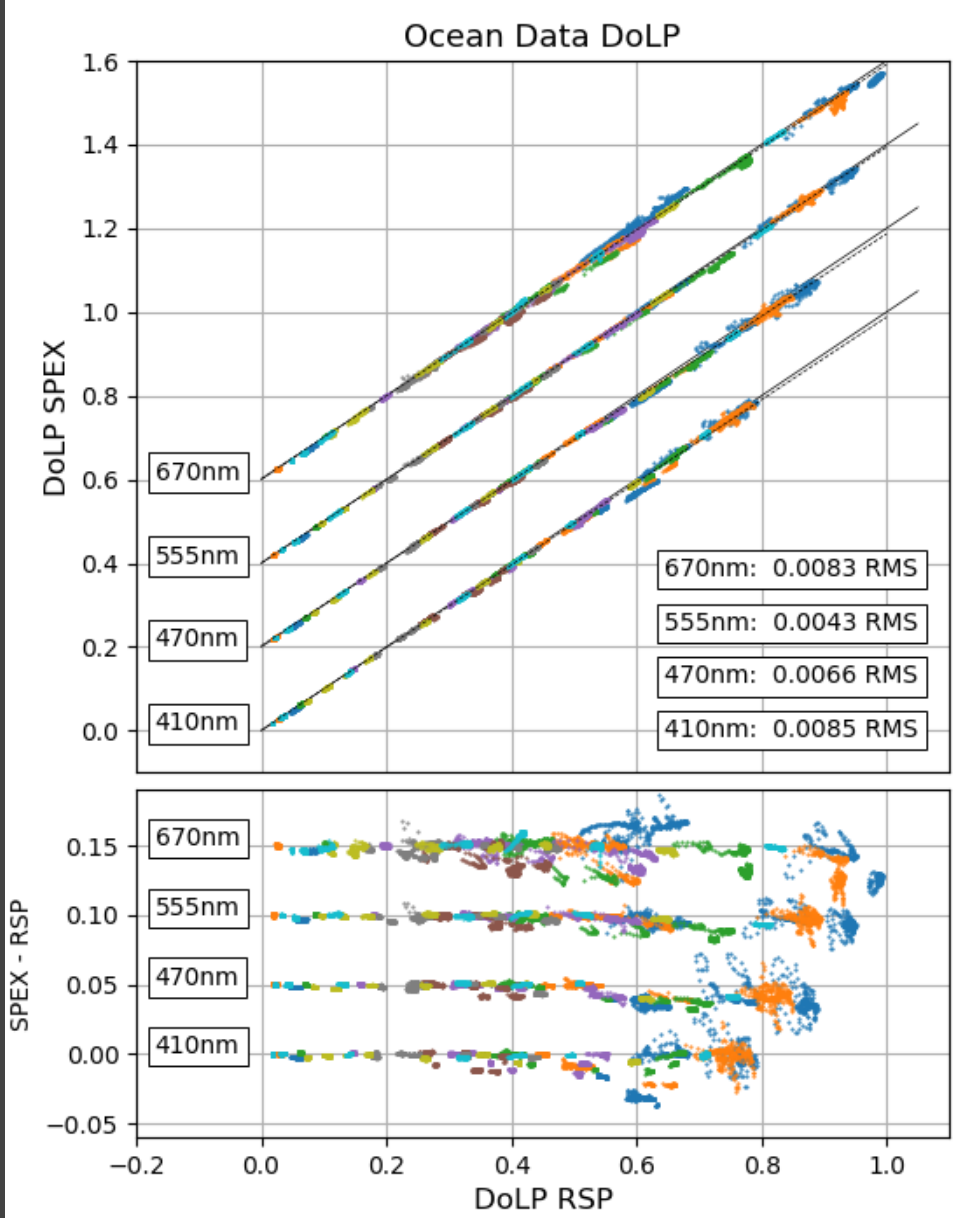

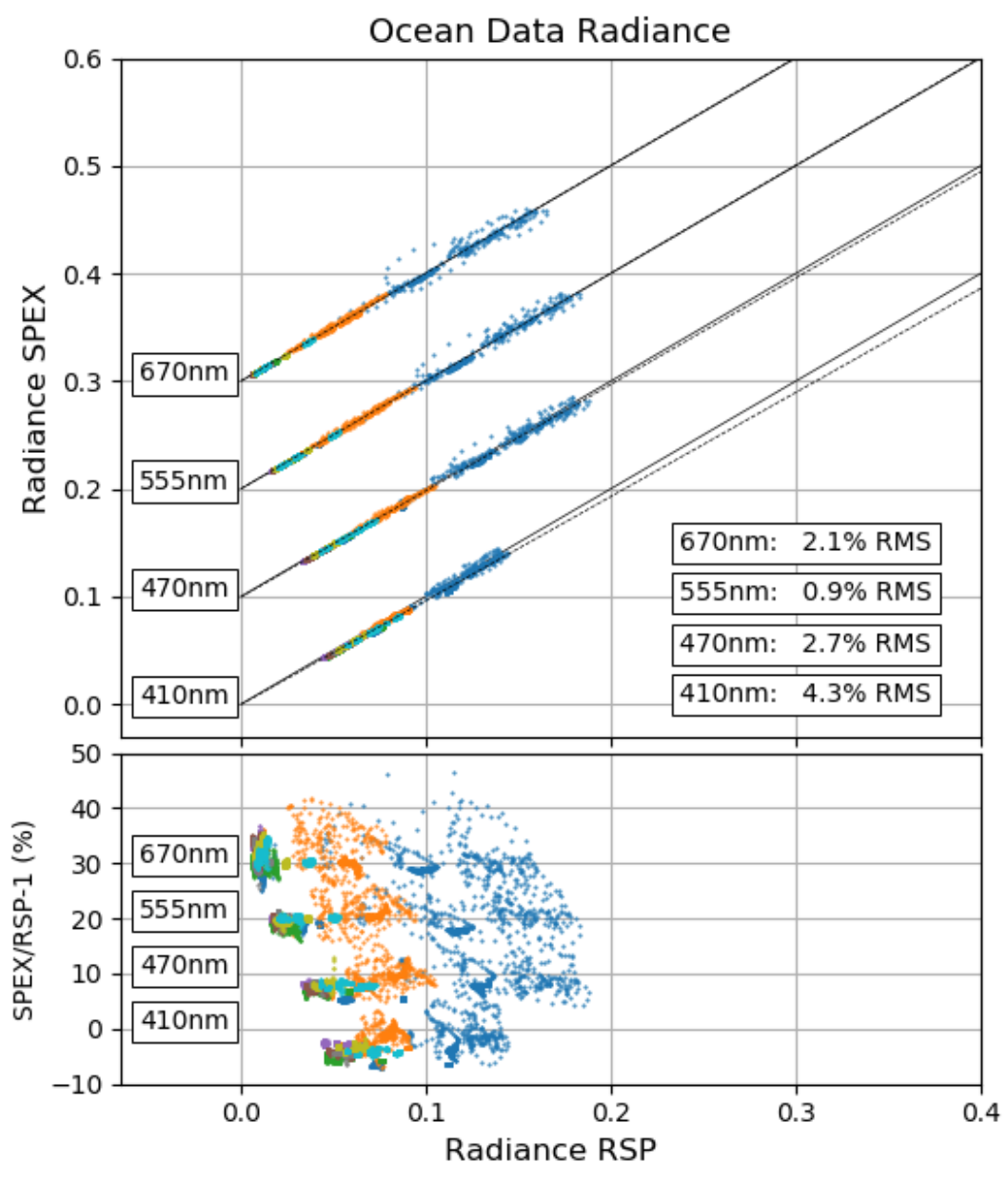




\section{SPEX airborne \& RSP over Clouds}
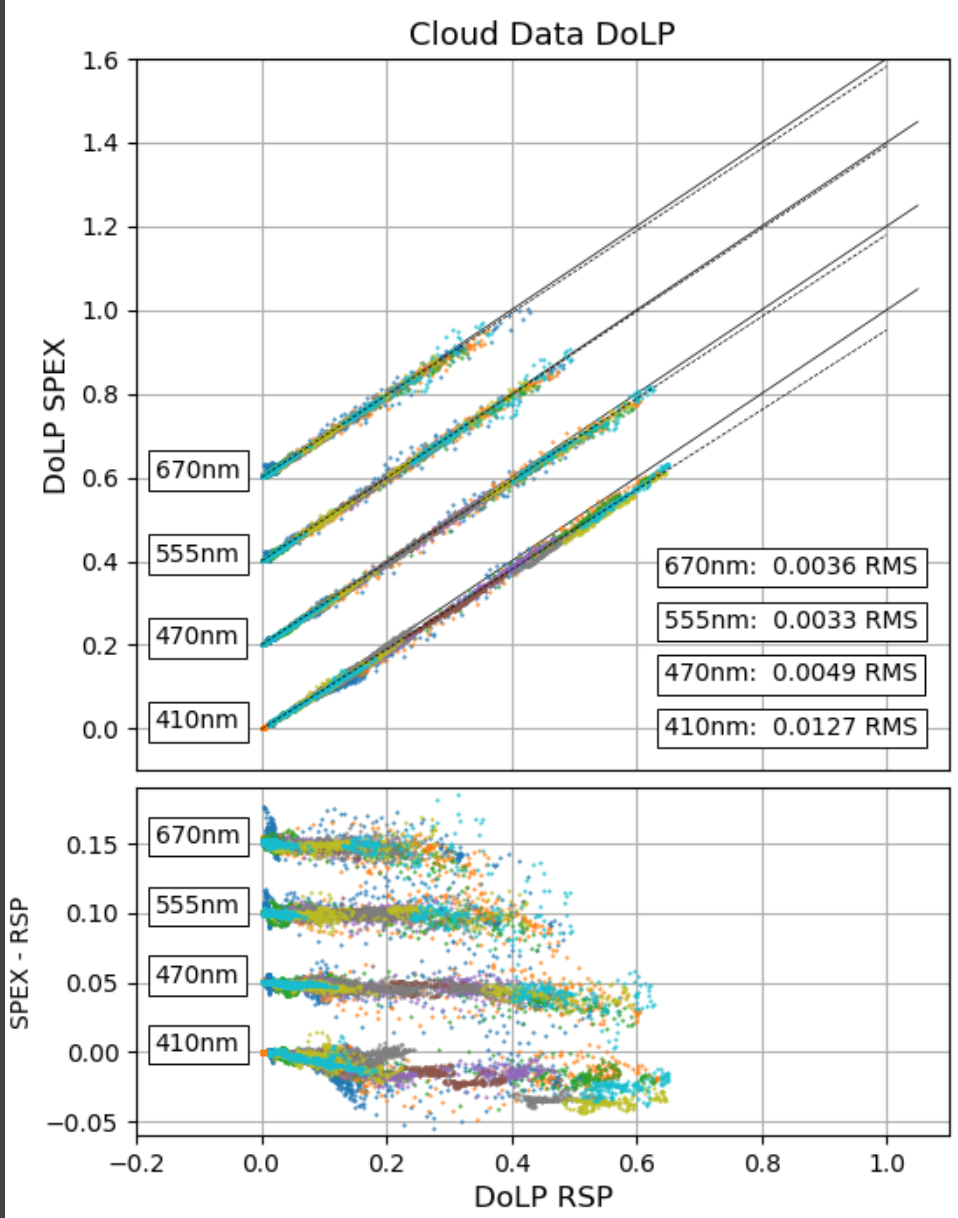

\section{Cloud Data Radiance}

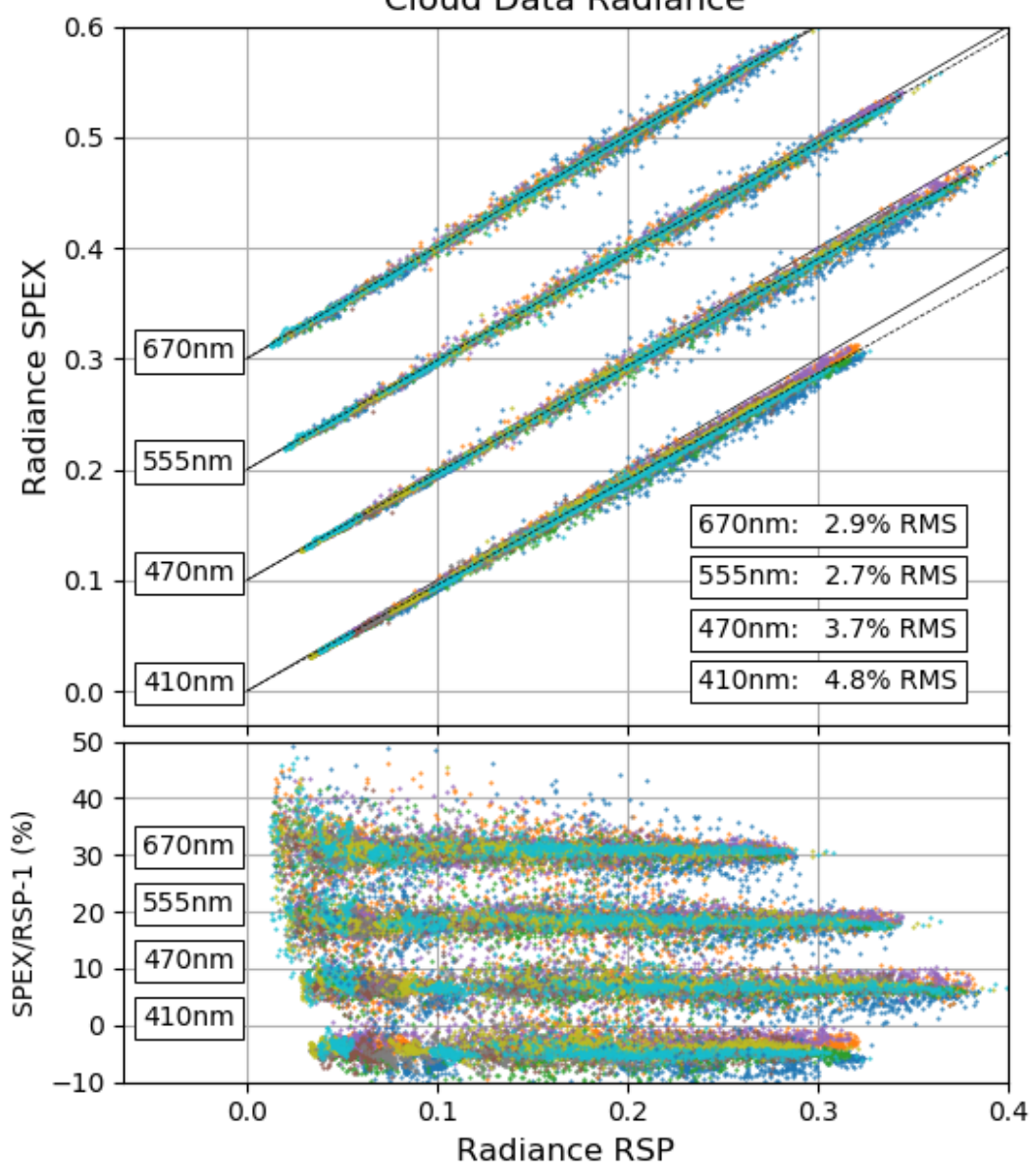




\section{SPEX airborne \& RSP over Land}
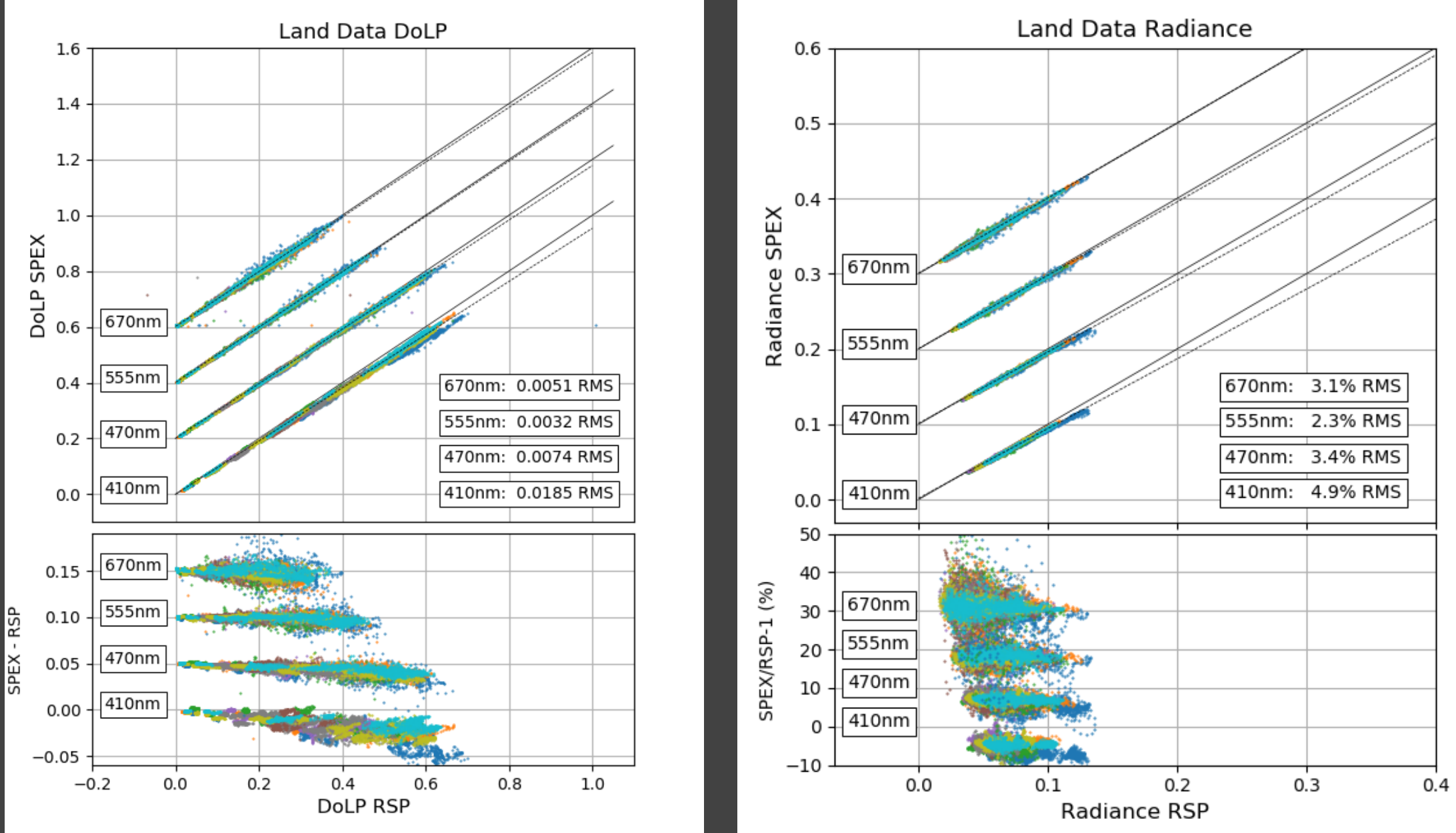

\section{SRON}




\section{Conclusions}

- In-flight Inter-comparison of SPEX airborne with RSP shows that SPEX airborne can deliver high quality, multi-angle polarization and radiance data

- Relevant for

- SPEXone instrument on PACE mission (2022)

- SCARBO $\mathrm{CO}_{2}$ mission concept study study, including

- SPEXone-like instrument

- SPEX airborne for flight campaign with Methane Mapper (MAMAP) and nanoCarb 